\title{
Capturing, Representing, and Interacting with Laughter
}

\author{
Kimiko Ryokai ${ }^{1}$, Elena Durán López ${ }^{2}$, Noura Howell ${ }^{1}$, Jon Gillick ${ }^{1}$, David Bamman ${ }^{1}$ \\ ${ }^{1}$ School of Information, ${ }^{2}$ Mechanical Engineering \\ University of California, Berkeley \\ Berkeley, CA, USA \\ \{kimiko, elena.duran, noura, jongillick, dbamman\}@berkeley.edu
}

\begin{abstract}
We investigate a speculative future in which we celebrate happiness by capturing laughter and representing it in tangible forms. We explored technologies for capturing naturally occurring laughter as well as various physical representations of it. For several weeks, our participants collected audio samples of everyday conversations with their loved ones. We processed those samples through a machine learning algorithm and shared the resulting tangible representations (e.g., physical containers and edible displays) with our participants. In collecting, listening to, interacting with, and sharing their laughter with loved ones, participants described both joy in preserving and interacting with laughter and tension in collecting it. This study revealed that the tangibility of laughter representations matters, especially its symbolism and material quality. We discuss design implications of giving permanent forms to laughter and consider the sound of laughter as a part of our personal past that we might seek to preserve and reflect upon.
\end{abstract}

\section{Author Keywords}

Laughter; tangible; containers; reflection; material quality; gifts; edibles; machine learning.

\section{ACM Classification Keywords}

H.5.m. Information interfaces and presentation: Miscellaneous.

\section{INTRODUCTION}

Laughter is an essential part of human social interaction $[20,28]$. Human laughter can elicit positive affect [2] and cooperative intent [28], has positive effects on health [4], and is considered socially contagious [20,6]. Laughter draws people together, tending to offer both physical relaxation and lowered communicative reserve [20]. Laughter is a fundamental, ubiquitous part of human life. Yet it is also ephemeral. We may immediately recognize the sound of someone laughing, but once the moment is over, the laughter is gone. The fleeting nature of laughter

Permission to make digital or hard copies of part or all of this work for personal or classroom use is granted without fee provided that copies are not made or distributed for profit or commercial advantage and that copies bear this notice and the full citation on the first page. Copyrights for thirdparty components of this work must be honored. For all other uses, contact the Owner/Author.

CHI 2018, April 21-26, 2018, Montreal, QC, Canada (C) 2018 Copyright is held by the owner/author(s). ACM ISBN 978-1-4503-5620-6/18/04. https://doi.org/10.1145/3173574.3173932 led us to consider ways to capture and share it in a concrete way. What might it mean to tangibly represent something so ephemeral as laughter? Might we treat our grandmother's laughter or our baby's giggle as if they were family heirlooms? Which laughs might we decide to keep and which might we let go? Might we gift our laughter to our loved ones? Over time, might it become a compelling way for us to think about when, how often, and in what contexts we laugh, or what laughter means to us? We consider laughter to be a part of our past that we might want to preserve and revisit, perhaps akin to revisiting old family photo albums or cherished keepsakes.

Starting with a vision of making laughter less ephemeral and considering it as something worth holding on to, two sets of questions drove this research. The first set of questions concerns the process of capturing laughter. What might it mean to capture our naturally occurring laughter over time, both technically and experientially? Many aspects of our daily life can already be tracked and quantified via a variety of activity sensors (e.g., walking, sleeping, stress, etc.). What might be gained by capturing and quantifying laughter like other dimensions of health and activity?

The second set of questions concerns representations of laughter and interaction with those representations. If our laughter can be captured reasonably well, what forms of representation should our laughter take? How might we interact with such representations, with whom, and in what contexts?

We conducted design research to explore these fledgling questions around capturing, representing, and interacting with laughter. We began with design explorations of laughter representations, which informed our initial ideas for physical containers and edible representations. Based on participant feedback, we refined our designs and

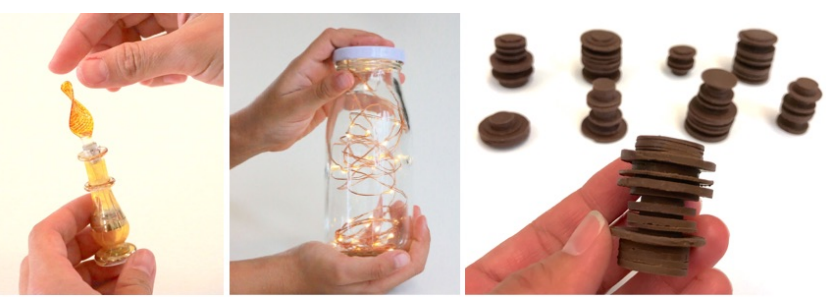

Figure 1. Left: A delicate bottle preserving laughter. Opening the lid releases the laughter inside. Middle: A jar preserving multiple instances of laughter. Right: Chocolate pieces representing quantity and quality (e.g., belly laugh, giggle) of laughter. 
implemented interactive prototypes for capturing and representing laughter. To represent laughter, we implemented both interactive containers (two types of glass bottles containing laughter) and boxes of chocolates, representing the quantity and quality of captured laughter. Participants could eat these chocolates while listening to their laughter. To probe interactions with these artifacts, we conducted a study in which participants recorded everyday conversations with their loved ones. We then extracted laughs from those conversations and converted them into the tangible forms mentioned above. Participants were invited to interact with and reflect on these representations of their laughter. We present our findings, discussing both implications of preserving laughter and positioning this topic as productive for the HCI community.

\section{BACKGROUND \& RELATED WORK}

HCI has explored a variety of ways we use technology to preserve and connect with past memories. Such studies address the ways we use physical and digital mementos for family remembering [e.g., 25, 5, 22] as well as the ways that interaction with digital mementos varies across generations, e.g., children [11], teenagers and college students [24, 3], and seniors [26]. Such memory objects are increasingly hybrid, blurring the boundary between physical and digital, and are often shared and gifted [30, 35] across a variety of contexts. HCI has also explored the gaps between how we perceive and treat physical vs. digital mementos. For example, in their study of digital gifting in comparison to conventional physical gifting, Kwon et al. [15] found that the experience of giving and receiving digital gifts lacks the most valued aspects of social gifting rituals: purposefully selecting an object; personalizing it; and thoughtfully giving it to the recipient. These deficits might ultimately undermine the value of the gifted object. Based on these findings, we leverage physicality and materiality to provide an opportunity to value and cherish representations of laughter.

Odom et al. [23] investigated why we preserve some things and discard others. Inspired by the design theorist PeterPaul Verbeek [32], they presented a three-factor framework to analyze what makes some things enduring while other things are easily discarded: 1) Function (what an object does), 2) Symbolism (what an object means), and 3) Material Qualities (what an object is made of and its sensual appeal). In their study, Odom et al. found that material qualities play an extremely important role in relationships characterized by a high strength of attachment between people and the object. We are particularly inspired by this work, as it helps us explore how people might perceive and value laughter were it given a concrete representation with material qualities. While laughter is an intimate part of our life, HCI has not yet considered laughter (especially the sound of laughter) as part of a past we might want to preserve and revisit. Building on Odom et al.'s framework of objects to be preserved or discarded, we explore the potential durability of laughter through our experimental, tangible representations.

\section{Technologies for Detecting Laughter}

HCI researchers have worked to detect and log laughter through a variety of sensor-rich, wearable instruments. For example, Hung et al. [9] presented techniques to detect different types of social actions - such as speaking, laughing, gesturing, drinking, or dancing - in a crowded social setting using a combination of a body-worn tri-axial accelerometer, an indoor positioning device, and a proximity sensor. Mancini et al. [18] presented a visionbased approach to detecting laughter by tracking users' head, shoulder, and trunk movements. Their system also required a wearable motion tracker. Laugh Log: E-textile Bellyband [29] is a corset with textile sensors designed to detect instances of laughter by looking at the pressure changes in the user's respiratory tract. Masai et al.'s AffectiveWear [19] is an eyewear with facial recognition system that is designed to recognize facial expressions (neutral, disgust, angry, smile, laugh, sad, and surprise). Lee et al. designed the Laughing Dress [16], a dress embedded with motion sensors and LEDs to explore how interactive wearable technology can support laughter as pro-social behavior in a public art installation. In contrast to these systems, our project aims to detect naturally occurring laughter without specialized wearable hardware, instead relying on audio capture from mobile phones that many people already carry with them.

In a related area, researchers have also explored smiling. HappinessCounter by Tsujita \& Rekimoto [31] is a set of appliances that encourages users to smile by detecting and providing feedback on users' smiles. For example, their mirror displays a happy or sad icon based on the presence or absence of smile, and their refrigerator only opens when a user smiles. Hernandez Rivera et al.'s Mood Meter [8], counted smiles in the wild and displayed the aggregated data in real time for the community to reflect upon.

Beyond recognizing naturally occurring laughter, our work also extracts that laughter from the surrounding conversation and preserves it for later listening. To our knowledge, this is original work in that we both automatically capture naturally occurring laughter and allow people to subsequently interact with it in tangible forms.

\section{DESIGN PROCESS}

We began our design exploration by brainstorming, sketching, and mocking up a variety of representations. In exploring representations of laughter, we grappled with questions around what constitutes a single laugh and considered variables such as a laugh's duration, frequency, and intensity. We thought about higher-level sound qualities such as those of a deep belly chuckle vs. a higher pitched giggle. Finally, we reflected on how very personal and unique the sound of each person's laugh can be, especially to their loved ones. 


\section{Containers: Laughter in a Bottle}

We studied a variety of physical objects to store and release precious laughter worth preserving. To elicit emotional engagement and create poetic and imaginative encounters, we avoided objects with existing associations to recorded laughter sounds such as a prankster "Laugh Bag" or a greeting card that plays a giggle or tune. Instead, we looked into cherished objects such as jewelry boxes, lockets, and perfume bottles that hold treasured items. Inspired by MusicBottles by Ishii et al. [10], we created two container concepts. The first is a small and delicate perfume bottle that holds and releases laughter. When the lid is removed, different instances of laughter are released, one at a time, via audio playback [Fig. 1, left]. The second container is a slightly larger jar that holds multiple instances of laughter represented as delicately flickering lights [Fig. 1, middle].

\section{Giftable Edibles}

Creating graphical visualizations of captured laughter data would have been a more traditional approach, but we explored tangible representations as a potentially more personal and poetic medium to interact with laughter. Moreover, we explored how such physical instantiations of laughter could be treated as objects that might be appropriate for gifting. Here, we explore materiality and physicality of laughter that could be considered giftable.

One playful and poetic way to gift laughter is to represent it as candies or chocolates [13, 33, 34]. Imagining a speculative future where we may have a candy or chocolate 3D printer on our kitchen counter, we created representations of laughter using edible materials such as candy or chocolate. Figures 2, 3, and 7 show our final design: a box of chocolates displaying laughter from 16 different events. The resulting edibles could be visually admired or casually eaten. Laughter is usually ephemeral and disposable. We intentionally designed this chocolate representation to be tangible but also perishable. We wanted to study how people decide to reflect on it or to simply eat it.

\section{Preliminary Design Study}

In a preliminary study, we invited 7 participants (age range between 20's to 60's) to hold our prototypes and imagine scenarios in which such objects could represent their history of laughter [27]. Participants found the idea of keeping laughter to be novel and imagined using such objects personally, capturing laughter of their children, parents, grandparents, siblings, and close friends. Most participants felt that sculpted chocolate might provide just enough resolution to reveal the "pattern," "rhythm," or "fabric" of their life (see details in [27]). While we received positive responses to our initial representations, these pilot participants could only imagine hearing their loved ones' laughter. In order to study people's experiences listening to their own laughter, we needed participants to collect their own laughter.

\section{Capturing Naturally Occurring Laughter}

Anticipating naturally occurring laughter and capturing a sufficient quantity of it turned out to be a challenging task, especially by manual means (e.g., hitting a record button on a phone right before laughter is about occur). As an exercise, the design team tried to capture laughter of our loved ones using mobile phones. We found this task to be difficult because people usually do not laugh on demand. We felt that the best way to capture naturally occurring laughter might be to have an extended sample of audio files and extract laughter from such recordings. To meet this need, we developed a machine learning algorithm to automatically detect laughter.

\section{FINAL DESIGN AND IMPLEMENTATION}

\section{Machine Learning Algorithm}

We implemented our laughter detection algorithm using a neural network that predicts, for every $10 \mathrm{~ms}$ audio frame in a file, an estimated probability that the given frame is part of a laugh. To make predictions, we looked at $750 \mathrm{~ms}$ of audio centered around a target frame [14], computed standard audio features (MFCC's and delta-MFCC's), and fed these inputs into a 3 layer feed-forward neural network. We trained our model on the Switchboard corpus, which contains approximately 260 hours of speech from about 2400 telephone conversations between 543 speakers [7], achieving $88 \%$ per-frame accuracy at identifying laughter in a held-out validation set from Switchboard.

In order to segment out the full duration of a laugh from a recording rather than individual frames, we used the low pass filter described in Kaushik et al. [12] as a postprocessing step. We also preprocessed the recordings from our participants with the Voice Activity Detector from Google's WebRTC to remove background noises and silence. The full algorithm takes in a single audio file and returns a list of audio clips in which laughs were detected. Our code and models are available for use on Github. https://github.com/jrgillick/laughter-detection

\section{Perfume Bottle and Jar with Lights}

We now detail the hardware of our tangible representations. The lids of the two bottle designs acted as binary switches. We embedded a mini speaker inside the perfume bottle and placed a string of lights inside the larger jar. An Arduino Uno R3 controlled the signals sent to the computer, which played the corresponding audio files or illuminated the lights in the jar.

\section{Boxes of Chocolates}

To quickly assemble edible representations of participants' laughter data, we created a system of buildable chocolates as well as a presentation box with embedded circuits to house the chocolates. Currently available chocolate 3D printers [such as, 1, 36] are good at making large chocolate sculptures, but their current resolution is not high enough to create small pieces of chocolate that would fit in a box for our purpose. In lieu of a printer, we devised our own design with chocolate building blocks [Figures 2, 3, \& 5]. Our first 


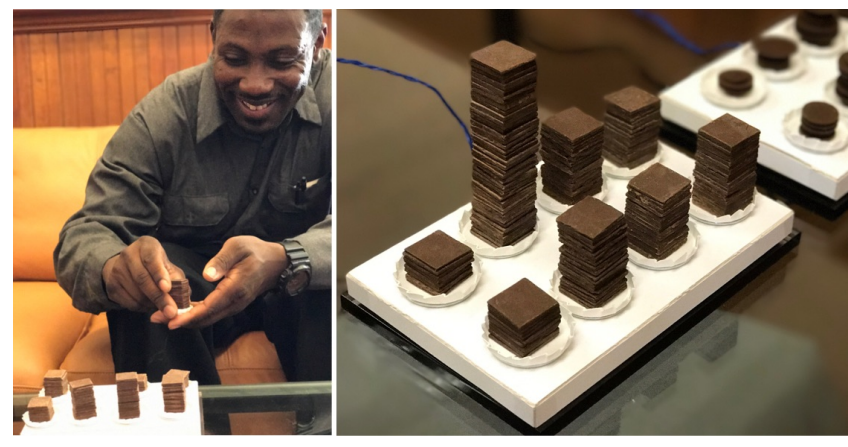

Figure 2. One box of chocolates was made of thin, square layers stacked up as towers. Each layer represents one laugh.

design involves thin, square layers stacked up as towers [Figure 2]. Each layer represents one laugh, regardless of that laugh's length or volume. The second design is based on thin, circular chocolate layers in three different sizes (large, medium, and small) representing a big laugh (e.g., belly laugh), a small laugh (e.g., giggle), or somewhere in the middle [Figure 3]. This process ended up being handprocessed, explained in the later section. In both designs, an event with many captured laugh results in a taller tower. The second design using chocolate circles can also reveal the relative quality of laughter (e.g., many giggles vs. a mixture of laughs). We assembled chocolate pieces by hand for each participant.
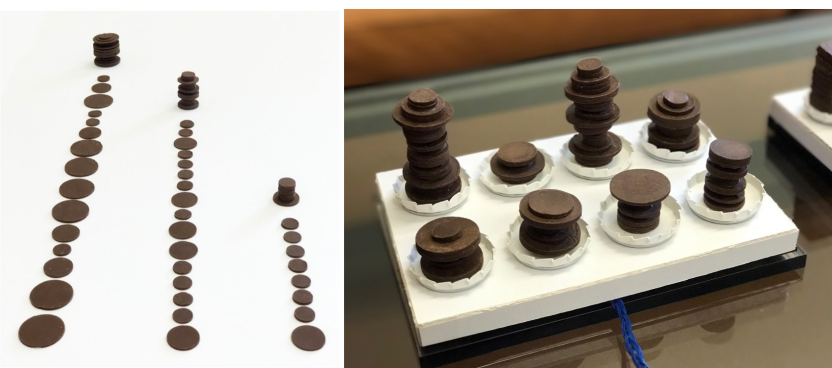

Figure 3. A second box of chocolate was made with thin circles of 3 different sizes. (large, medium, and small) representing a big laugh (e.g., belly laugh), a small laugh (e.g., giggle), or somewhere in the middle. Left: An example of how three different towers were made from a series of different sized circles. Right: A box of chocolates made from the circular chocolate layers.

The chocolate pieces were displayed to participants in a special box wired with conductive thread and copper tape, with the latter serving as an interface between the soft and hard connections in the circuit. Each chocolate piece sat on a paper dish with a metal bottom that functioned as a switch. When a chocolate was picked up, it signaled the system to play the laughter associated with that particular tower.

\section{STUDY}

In total, 20 people participated in our study. We originally recruited 18 participants (10 females, 8 males). Two participants (both male) dropped out, as they were unable to complete their recording assignment within the 8-week study period. After completing the first study, 4 participants returned to repeat the experience with a loved one. We discuss this second part of the study in a later section.

In our first introductory meeting with participants, we briefly described our algorithm to extract laughter from audio recordings and invited participants to collect their own audio files containing naturally occurring laughter. We asked the participants to collect about 3 hours of audio across 18 separate recordings (approximately 18 ten-minute long files, although some files were as short as 5 minutes while others were as long as 20 minutes). We invited participants to think about different occasions, people, and contexts for laughter instead of providing a single 3-hour long file from one event.

We assured participants that we would not transcribe or analyze any of their recorded conversations. Only the laughter files resulting from the extraction algorithm would be analyzed. In accordance with our approved IRB protocol, participants were asked to inform all conversation partners that the recordings were for academic research and to obtain verbal consent prior to recording.

All participants were initially given one week to collect the 3 hours of audio and upload the files in a secure online folder. About one third of the participants finished their collection within this target period. The remaining twothirds spent several weeks collecting the 18 files. Once participants' audio files were uploaded and processed, we scheduled one-hour-long individual meetings to discuss the results of their collection. In each individual meeting, the participants were presented with the tangible representations. We displayed these representations in the following order: 1) perfume bottle, 2) jar, 3) chocolate with squares, and 4) chocolate with variable circles. We asked participants about these representations and also about their audio file collection process.

\section{RESULTS}

\section{Listening to Personal Laughter}

Interviews with participants revealed that their audio files contained laughter from a variety of people in their lives including girlfriends, boyfriends, wives, husbands, children, mothers, fathers, siblings, friends, professional colleagues, and roommates. The context for laughter varied from cuddling in bed to family dinner to professional meetings to field trips.

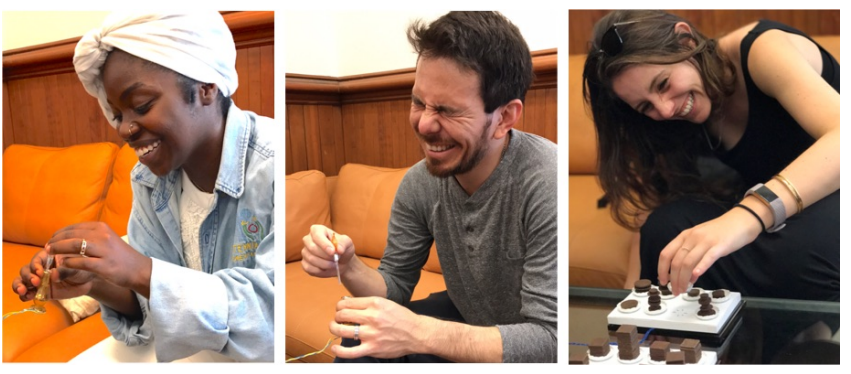

Figure 4. Participants listening to their loved ones' laughter. Left and Middle: Participants with the perfume bottle. Right: Participant with chocolate pieces. 
When the participants heard laughter of their loved ones through our tangible representations, they responded in delight with awe and smiles, and in some cases, even strong bursts of laughs that brought tears to their eyes [Figure 4]. Listening to her children's laughter, Zooey smiled and exclaimed, "It is extraordinary!" John, who heard his girlfriend's laughter in the perfume bottle, smiled and said, "This makes me feel emotional. Even though, I just saw her 10 minutes ago in my apartment, this makes me miss her." He said he was surprised how listening to his girlfriend's laughter in the bottle made him feel "tender" inside. Matt heard the laughter of his close friend and said, "It's made me very happy since I've been here (interview room). Usually I don't feel myself smiling, but I feel myself with a big one of these (pointing at his smile) right now, which I don't do as much, only when I'm with my close friends. This is bringing me back to few of those times, which is cool." Mary heard her friend's laughter and said, "I didn't expect it to be so moving. He is just my school friend, but I was genuinely moved by his laughter."

The participants showed great care in listening to the details of laughter. Denise heard her father's laughter and said that it represents "the best part of the conversation." She said the experience of listening to his laughter made her realize "how precious moments of laughter really are." Such reflection on laughter led many participants to mention the therapeutic aspect of listening to laughter. In response to our question about when they might listen to the laughter, Johanna said, "I would listen to this when I am feeling sad or lonely." Mary said, "On days when I wake up on the wrong side of the bed, or not feeling so great with a feeling of despair, I would use it (pointing at the bottle) to pick me up." Perhaps, listening to individual laughter removed from its context gave them an opportunity to focus and reflect on the positive aspects of their memory.

\section{Representations of Laughter}

After introducing participants to each representation and inviting them to interact with it, we asked participants to rank the representations according to preference. Building on Odom et al.'s framework, we evaluated our designs in terms of their function, symbolism, and material qualities.

Perfume Bottle and Jar with Lights: Most participants highly ranked the perfume bottle and jar.

Function: The object's function was clear to all participants. Opening the lid released its contents - the laughter - and closing the lid preserved the laughter inside. Eric said, "the laughter is trapped inside, and I love it." Zooey said, "If only we could bottle our laughter and gift it to our loved ones. Now we can with this!" Derek said, "I'll treat myself with a dose of laughter each day (gesturing opening and closing of the bottle)." Many also liked the small size and mentioned the possibility of carrying the bottle with them.

Symbolism: Without any prompt from us, several described how listening to laughter from bottles was like "letting the genie out of a bottle," especially for the smaller perfume bottle. In listening to her daughter's laughter in the bottle, Cindy exclaimed, "That's Melanie in the bottle!" Many participants also noted the association between delicately flickering lights inside the jar and the "laughter lighting up our life." The perfume bottle, even though it did not contain any fragrances, evoked olfactory sensations with some participants. Four participants expressed that they detected fragrance as they opened the lid and listened to the laughter. In addition, 5 more participants made an association with "aroma therapy." For example, Layla said that she would keep her boyfriend's cologne together with his laughter.

Material quality: The participants reacted to the material quality of the glass bottles. In particular, the perfume bottle with its minute glass details was perceived as precious and delicate. Camille felt the "cuteness" was particularly well fitting in preserving the laughter of her 4-year-old daughter. For most participants, the bottles' preciousness and delicacy led them to feel that this was an object for personal and private use (i.e., "only for me") as opposed to an object to be shared with friends. They also said that it should be kept in a safe place such as in their bedroom, on the nightstand, or in a cupboard.

Overall, the bottles appealed to the participants in all three factors. As Odom et al. found, the mutually reinforcing interrelations among function, symbolism, and material qualities seemed to contribute to their high rating.

Chocolates: Chocolates also ranked high in participants' preference. After picking up and listening to laughter embodied in chocolates, the participants were invited (but not required) to eat the chocolate. All participants willingly tried the chocolates, and after consuming some chocolate, they were asked to share how they felt eating the representations of their laughter.
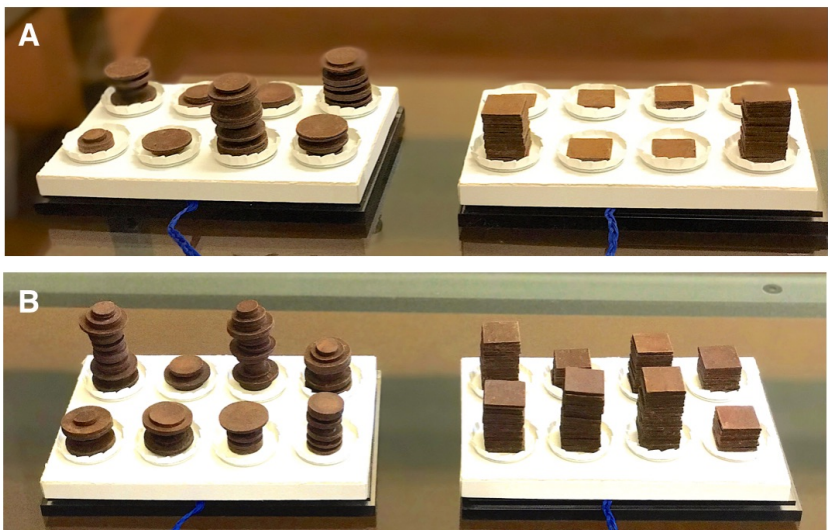

Figure 5. Top (A): Captured laughter of one participant. Bottom

(B): Another participant's captured laughter. Participants did not see each other's chocolates, but in this figure, one can see differences in the quality and quantity of laughter.

Function: While each participant expressed varying degrees of fondness towards chocolate and sweets in general, nearly all enjoyed the experience of tasting the chocolate while 
listening to laughter. Almost all mentioned the layered happiness, i.e., enjoying the taste of chocolate and hearing laughter simultaneously, appealing to multiple senses. Matt said, "I enjoyed the combo of eating and listening to it. It gave me a few more senses, sensory of taste, sweetness, and the past event I'm listening to it. Feeling the texture of (chocolate as) something that you've experienced was interesting." Most expressed that the chocolate tasted sweeter than they expected. Zooey explained, "You are already happy listening to the laughter, and you double it with the sweetness of chocolate." Two participants, however, expressed a disconnect between listening to the laugher and eating the chocolates. Elizabeth preferred to eat chocolate only after listening to laughter because she wanted to focus on the auditory experience first. Eric similarly explained that for him, "the laughter is dominant, and eating chocolate is the second." He said that eating the chocolate was somewhat distracting while he was trying to focus on the laughter. These reactions raise an interesting design challenge of engaging people in reminiscing through multiple senses. Most participants enjoyed engaging via not only the tactile and auditory but also taste and olfactory senses; however some preferred to focus on listening alone.

In general, the chocolate towers showed the relative quantity and quality of their captured laughter. Yet, overall, the display of quantity did not seem salient to participants. In other words, participants did not seem to care so about how much they laughed. For example, some participants commented that some of the tall chocolates were "intimidating" (i.e., too big to eat) [Figure 5], while others went for the biggest pieces in order to get more chocolate. In terms of the chocolate's function as reflecting the quantity and quality of laughter, the unevenness of the chocolate layers compromised the fidelity of the resulting towers. For example, a chocolate tower with 22 layers to represent 22 instances of laughter was sometimes be as tall as 20 layers or 24 layers because of the inconsistent thickness of the handcrafted layers. The chocolate towers with varying circles were also often misunderstood. Participants often thought that the towers represented varying amplitudes of the sound profile of the entire audio file, while it actually represented each laughter instance and its relative loudness. When this misinterpretation arose, we explained the correct mapping, but these distinctions did not seem to matter. Most participants still highly ranked the circle towers.

Symbolism: In contrast to the perfume bottle and jar, which were more personal and private, participants felt that the chocolates were for gifting and sharing. Participants explained that they would choose to share or show off the chocolates with family and close friends. Despite some misunderstandings of what the different shapes represented, most participants preferred the towers with varying circles over squares because they were "visually more interesting" and had "more meaning behind it."
Material quality: While most participants reacted to the consumable material quality of chocolate by eating them, some reacted to its ephemerality and hesitated to eat more chocolate pieces. Denise said, "It's very illogical. Not wanting to throw away something you have recorded. Even though laughter gets thrown away all the time." As she held the chocolate pieces, she called them "delicate beautiful food." She said, "I was raised in a blue-collar worker family so food was precious." So if the food had a particularly intricate shape, she was less inclined to destroy it by eating it. The decision whether to eat the chocolate representations was further complicated for Denise by a mismatch between the seemingly more complicated shape (which in her view deserved more admiration) and the actual laughter it represented. As she respectfully held a large piece of chocolate with complicated shape, Denise said, "This one is not a particularly special moment or anything. This is kind of social throwaway laughter, nothing that sentimental. But it looks visually beautiful." On the other hand, the "best piece" for Denise that included her father's laugh, was a shorter and less complicated shape, which was equally beautiful to her.

Six participants explicitly commented on the actual taste of chocolate, such as, "This is a very high quality chocolate!" More interestingly, although all chocolate pieces were made with the same dark chocolate, three participants thought that we used different flavors for different pieces (e.g., commenting, "This one is nuttier than the other," "This one is sweeter than the other one"). Overall, the chocolate did not seem to have high function. Still, high symbolism and material qualities seem to contribute to the participants' preference towards the chocolate laughter representations.

\section{Reflections on capturing laughter}

We asked participants whether it was easy or difficult to collect the requested 3 hours of audio samples. Five out of 16 participants said it was easy and completed the collection on time. However, the remaining 11 participants indicated that the process was rather difficult and needed 34 additional weeks to complete their collection. Participants described multiple levels of difficulty, the first of which was remembering to record. Many participants expressed frustration that they often forgot to record or wished they had recorded a particularly laughterful conversation. A second issue arose from privacy concerns. Many participants explained that their friends were apprehensive about being recorded even for academic research purposes. Some participants also mentioned that they accidentally captured arguments or unsavory conversations and decided to delete some of their recordings, making it difficult to reach their collection goal. Finally, there were technical constraints. Our laughter detection algorithm did not work on recordings with significant background noises. Participants found this requirement constraining as they explained that natural laughter tended to occur in noisy environments (e.g., during a commute, parties, mealtime at restaurants). These issues prompted additional questions 
about future technical work, as well as whether the laughter capturing process can be automatic or manual.

\section{Should laughter be collected automatically or manually?}

Given the difficulties of collecting laughter over multiple weeks, we asked the participants whether they could imagine using a household device such as Amazon's Alexa to automatically collect laughter in the future. Participants who felt that their personal experience of manually collecting laughter was arduous (11 out of 16 participants) tended to prefer future automation. Participants who found manual capture of laughter to be easy were split between preferring automatic vs. manual capture. Despite the work involved, a few participants said they would still choose to record manually for privacy and security reasons. Two participants explained that they would be OK with automatic capture if it happened without connecting to the Internet.

Participants who opted for automatic capture described several anticipated advantages. First, they expected to feel more natural while their laughter is being recorded, as they wouldn't have to think about it. Many described an initial un-naturalness in having to manually reach for the phone to record and in their conversation partners' reaction to it. In addition, participants said that automatic recording would provide a sense of surprise and whimsy. Allison mentioned that she would not mind getting prompts such as, "On this day last year..." or "Remember this?" as Facebook and Google currently do, but with her laughter as the content. For some participants, automation might mean easing up on the responsibility. Denise, who felt ashamed of capturing awkward laughs in response to an inappropriate joke, said, "If the machine did it, I would feel less responsible for it." For some, the decision to choose automatic or manual capture depended on whose laughter was being recorded. For example, Camille wanted her toddler's laughter to be captured automatically, but for gifts for her friend, she said wanted to collect it manually so as to have more control.

In a future system, the ability to decide whether to record manually or automatically according to user preference and context will be important.

\section{Which laughter to keep and which to let go?}

Assuming that we amass a large collection of laughter over time, we asked participants how they might decide which laughter to preserve and which laughter to discard. In their responses, the participants described the "nuances" of laughter. For example, Johanna said, "Not all laughs are the same" so she wished to keep only the "natural laughter." Similarly, Denise said that she finds her herself using laughter as a social tool, such as fake laughter in social settings to save face. Detecting differences between genuine and fake laughter is beyond the scope of the current project but is an interesting question for the future technology.

Melanie said that she would like to control whose laughter she listens to. Using such a filter, she could "choose to listen to laughter of only this person but not that person, etc." Relatedly, many participants preferred not to listen to their own laughter. Interestingly, they did not mind listening to their own laughter if it was mixed with their loved ones' laughter. Future designs could include a mechanism to selectively record laughter of particular subjects, as in "record only my daughter and my husband." Furthermore, deciding which person's laughter to keep creates technical opportunities for long-term identification and preservation of unique audio other than laughter.

\section{Not all laughter is joyful}

While participants responded positively to most laughs in our prototypes, a few participants had laughs that they would rather silence. For example, Denise found a couple of instances of laughter she was embarrassed about, later explaining that the laughter followed inappropriate jokes. When she encountered such embarrassing laughter in the chocolate, she said she was "more in the rush to eat it and finish it" (rather than savor it). This response was particularly interesting as she contrasted it with another piece that she "didn't want it to end (hearing of the laughter)" and even "wanted it to extend." On the other hand, Denise said that the experience of "silencing the laughter by eating it" was "gratifying, snapping down on something crunchy." The function of chocolate in this case added to the removal of the laughter, "accomplishing something by getting rid of it." Savoring the memory versus crunching down to eliminating memory is another interesting design opportunity.

\section{Private laughter}

Our prototypes contained participants' laughter and that of their loved ones without any identifiable parts of their original conversations. Yet, our participants could easily discern which laughter in the prototype belonged to which person in their life. They also noticed how private their laughter felt. For example, Melanie noticed, "My laughter changes in front of people who I know well or not." Many participants, therefore, felt their laughter is so personal that it should not be shared outside of their close friends and family. For example, Matt said that he would not want laughter to be quantified and displayed in social media, in the way "Likes" are quantified and shown on Facebook. Participants wanted the representation to reflect the level of intimacy and privacy they felt in their laughter.

\section{Tangibility of laughter}

Daniel had a piece of chocolate that consisted of entirely his own laughter because he had recorded himself watching a funny show alone. He listened to his own laughter and he initially ate only half of the piece because it was quite a big piece containing 18 layers. We later asked him what if we ate that piece instead of him? Daniel seemed apprehensive and did not want us to eat the piece. He explained that if someone other than him ate that piece of chocolate, "It would definitely feel like invasion of privacy. It feels weird to feed it to another person. It feels strange because it is part of me, reflection of me. Even if it's my girlfriend, it is 
strange. It's like if it's not your laughter, you do not have the right to eat it! Only I should be able to eat my own laughter. Especially, if it's a laugh of single person.” Right before he left the interview room, he grabbed the last bit of uneaten chocolate and popped it into his mouth. He said with a smile, "I'll eat it so no one else can eat it!"

Daniel's reaction might be explained by Mary's account. Mary said that she takes voice notes all the time, but she feels audio files are de-personalized. In describing why tangibility of the laughter representation mattered, she said, "Having something to touch, or something to eat helps it reconnect with what was de-personalized." She further described that the tangible representations of laughter "should not be disrespectful or throwaway." In a way, Mary is describing the augmentation or ensoulment [24, 21] of an object with laughter. By leaving our body, our laughter becomes disembodied. By re-encountering laughter as a concrete embodiment, we are reunited with our laughter. Thus, for Daniel, the representation felt as if it were "part of me" or "reflection of me" that should only be consumed by me and is not open to public. Thus, tangibility of laughter representation matters in respectfully reconnecting what was once part of us.

\section{Evaluation of automatic laughter detection algorithm}

Our automatic laughter detection worked fairly well as long as the audio file did not contain much background noise and the person was relatively close to the microphone. For example, in our own evaluation of sample audio files containing laughter, approximately $90 \%$ (9 out of 10 ) of the laughter instances was accurately detected from clean recordings without any background. However, when the files did not meet such requirements, the algorithm would return 10 times more instances of laughter than the actual count of laughter. For example, noises such as nocks on the table or wind blowing were misinterpreted as laughter (false positives). We informed our participants of this technical constraint, asking them to record audio with no background noises and to have their partners close to the microphone. This request was difficult for many participants. Participants' effort to have the microphone close to their partners led them to, for example, carry the phone in their pocket and as a consequence, inadvertently create background noise as the phone rubbed on their clothing. Overall, participants' files were noisier than we hoped for. As a consequence, we manually post-processed many of the files, including designation of the laughter quality (loud, soft, or medium). We originally had a script that ranked loudness of each detected laugh but were unable to utilize the script due to the unanticipated low audio quality. Additionally, we asked a few participants to resubmit recordings of higher quality.

\section{Giving it another try}

We wanted to give the three different types of representations - jars, chocolate, and graphs - equal chance for consideration by all participants. Therefore, we did not reveal in advance how their laughter would be represented. Towards the end of the interview, we asked participants if they would have recorded their laughter differently. Seven out of 16 participants answered that they would collect the laughter of a specific person exclusively (e.g., best friend, mom, girlfriend) and would be more intentional about recording (e.g., selecting occasions to commemorate). Of those 7 participants, 5 agreed to continue recording with a selected partner and to return for another interview with that partner. While the original participants were familiar with the tangible representations, their partner would remain unaware, to be surprised by the representations as gifts during the final interview. Since 1 of these 5 participants had unexpected travel during our study period, we had 4 pairs of participants for the second round.

\section{SHARING \& GIFTING LAUGHTER: SECOND ROUND}

Social reflection vs. personal reflection

The recipients of a laughter gift were surprised to hear their laughter from the representations. Learning that their partner collected laughter over time to make these gifts made them feel humbled and tickled. They smiled, hugged, and laughed together in response to hearing their laughter [Fig. 6]. As they listened to different laughter, participants reminded each other of places and occasions where it was recorded, such as "That's from my birthday party!" "Remember where we were?" "Oh yeah, I remember lying on the grass with you." Melanie, who recorded her mom exclusively for this second round, enjoyed sharing her laughter collection with her mom. She said, "That's how I wished I could share. Like a game or puzzle of shared
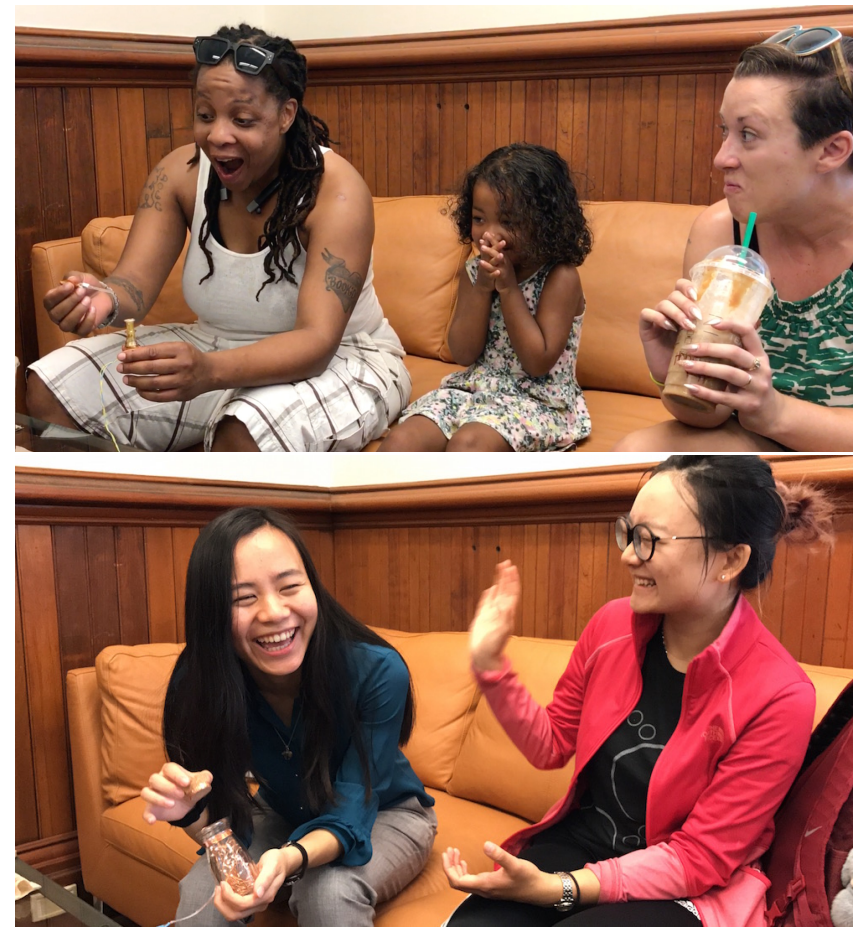

Figure 6. Participants share captured laughter with loved ones. Top: Participants with the perfume bottle. Bottom: Participants with the jar. 
memory." Melanie said the collection of laughter had "the right amount of recency. It's just last week (pointing at the laughter from her collection) but we start to forget the details. If the laughter was from too long ago, it almost becomes meaningless." But her mom Cindy had different feeling towards her own experience. She responded to one of Melanie's big laughs and said, "When I heard her laughing, it brought me back to the time when she was a baby. She would have these big belly laughs. It was so adorable. And you still sound that way. [Melanie and Cindy laugh together.] When I just heard that laugh, it was very sweet." So Cindy wanted to have a bottle with baby Melanie's laughter. Melanie thought the idea of having a container with her baby laughter was sweet, but it was more for her mom than for her. For Melanie, the experience of reflecting with laughter was more social. She said, "It is more meaningful to have it with someone who can share. Between two of us, or a number of us. The more you experience the memory together, the more you remember, so it cements it." Thus for Melanie, recency mattered. Yet for Cindy, the idea of baby laughter was not about recency but rather quite the opposite, as more of a time-capsule. Reflection with laughter therefore should be supported as both a personal and social activity.

\section{Permission to record}

All our participants received verbal consent from their partners prior to recording them. Most of the partners who were recorded appreciated being informed. Cindy said that it would have made her feel like she was "a bad mom" if her daughter was recording her voice without her knowing. Tess said that she would not have minded the gift as a surprise even if she hadn't been asked for consent. She said that being asked to record made her feel some "pressure" to produce good laughs. Layla said it felt slightly unnatural that John started to record and that they had to hangout subsequently knowing that the recording has started. Layla described that once the consent was given, she would not have minded the recording to take place automatically. This illustrates that informing our loved ones about recording and getting consent is mandatory. But starting the recording automatically with or without letting the partner know could be a separate design issue.

\section{DISCUSSIONS}

\section{Ephemeral laughter and permanent representation}

We started with the vision of making laughter less ephemeral and giving people opportunities to interact with concrete representations of their laughter. Some of our representations (e.g., bottles) were more permanent than others (e.g., chocolates). The difference in perceived material qualities between these representations revealed that people do care about how enduring the representations are. Given that laughter is usually fleeting, we originally felt that non-permanent representations such as chocolates might be more appropriate than more permanent representations such as wood sculptures. Yet, ephemeral representations such as edibles made some of our participants nervous. Perhaps, one of the key findings of our study is the ensoulment [24,21] of the objects when it is augmented with laughter. Once laughter is made concrete in an object, what was once disembodied becomes embodied again in the object. Accordingly, the participants regarded the representations as "part of me" or "reflection of me" that should not be discarded or should "only consumed by me." Designers of laughter representations must be sensitive to the intimate quality of this work.

\section{What reflection with laughter is not about}

We also began to reveal what people felt laughter reflection is not about. Initially, we imagined that people might want to celebrate moments of their life when they laughed a lot, like an accomplishment. However, our study showed that more laughter did not always mean more memorable or happier moments. To our surprise, the participants did not seem to care so about how much they laughed. Instead, they cared about whether or not the representations contained laughter that reminded them of their happy, lived moments with their loved one, regardless of quantity. By making concrete embodiments of laughter, we were able to reveal that the quantity of laughter is not likely to become the focus of laughter reflection.

\section{Why is this an $\mathrm{HCl}$ topic?}

While people's oral stories and environmental sound have been studied in the context of remembering [e.g., 25, 5, 22], laughter, in its own right, has not yet been considered within the HCI community. Increasingly, many aspects of our lives are collected, analyzed, and reflected upon. We predict that, in the near future, laughter will also be captured, and HCI researchers will need to address questions about what to do with that captured laughter. Our study begins to illustrate that people consider reflection on laughter to be both a personal and social activity. While they may reflect on it alone or with loved ones, people felt that laughter is something so personal that it should not be shared publically. Therefore, we need to be careful about designing laughter embodiments that could be shared in person but might be equally suitable for safekeeping and private use. Furthermore, issues of privacy made the process of capturing laughter challenging for many of our participants. More study is needed to better understand the issues of privacy and security in capturing naturally occurring laughter. We believe this research builds on many research efforts within HCI ranging from collecting and storing of personal information to the design of embodied tangible interactions for something as ineffable as laughter.

\section{FUTURE WORK}

Our participants often found themselves in noisy environments while trying to capture naturally occurring laughter. In order to make a truly automatic laughter capturing system, the algorithm must be able to work with a variety of background noises. To improve its usability in the wild, the future system might augment existing datasets by overlaying training audio with ambient sounds to simulate laughter in a variety of contexts. 
Background noise also played an interesting role by reminding participants of their laughter's context. Some sounds (e.g., background music from a restaurant, water sounds at the pool, snippets of conversation around the laughter) were included in individual laughter segments. We asked participants about preserving context, and most enjoyed listening carefully to the laughter itself. When snippets of conversation were included in laughter (e.g., from an overlapping conversation), most participants found it distracting. A few participants indicated that a bit of context might help them better remember the conversation. Originally, we had considered expanding the duration of laughter snippets, e.g., preserving a few seconds of audio that precedes (or follows) the laughter. However, we chose to only record laughter for this study to 1) mitigate privacy concerns of sharing potentially sensitive conversation topics and 2) encourage people to focus on and listen to the nuance and meaning of laughter itself. We see the question of how much context to include as delicate balance: too much would detract from the laughter, making the recordings more about the conversation than the laughter. Providing customizable settings might be a solution for the future system.

We also consider our design's long-term implications. Our original approach was to create opportunities to cherish fleeting laughter in ways that transcend time and context. In light of participant feedback, we consider automatically archiving contextual information in the backend so that when context recall is desired, simple cues can be provided. Examples include a time or location stamp on the bottom of the bottle or box (like an aged stock) or reintegrating conversational context into the audio clips. We do not envision the physical representation overtly displaying the contextual information but rather serving as a pointer to a digital archive.

\section{CONCLUSION}

We demonstrated that laughter can be given enduring forms and play a role in people's reflection on past memory both in personal and social contexts. This work contributes a qualitative evaluation of our prototype system for capturing, representing, and interacting with laughter. Our study showed that the tangibility of a laughter representation matters, as it serves to reconstrue what was once disembodied into a concrete reminder of a happy moment that people can preserve or share with others. We also found that people valued the ensoulment of objects with their loved ones' laughter, not the quantity of laughter itself. We offered design insights for considering the sound of laughter as a part of our past we would want to preserve and reflect upon.

\section{ACKNOWLEDGMENTS}

We thank all of our participants for their time and invaluable feedback. Our special thanks go to Leah Rosenbaum for all her wonderful help. We also thank Dina Bseiso and Ji Won Jun for their discussions.

\section{REFERENCES}

1. Gosse Adema. 2015. 3D Chocolate Printer (made from LEGO). Instructables. Retrieved March 19, 2017 from $\mathrm{http}: / / \mathrm{www}$. instructables.com/id/3D-Chocolate-Printermade-from-LEGO-1/

2. Jo-Anne Bachorowski and Michael J. Owren. 2001. Not All Laughs are Alike: Voiced but Not Unvoiced Laughter Readily Elicits Positive Affect. Psychological Science 12, 3: 252-257.

3. Elizabeth S. Bales and Siân Lindley. 2013. Supporting a sense of connectedness: meaningful things in the lives of new university students. In Proceedings of the 2013 conference on Computer supported cooperative work (CSCW '13). ACM, New York, NY, USA, 11371146. DOI: https://doi.org/10.1145/2441776.2441905

4. Gregory A. Bryant and C. Athena Aktipis. 2014. The animal nature of spontaneous human laughter. Evolution and Human Behavior 35, 4: 327-335. http://doi.org/10.1016/j.evolhumbehav.2014.03.003

5. Lina Dib, Daniela Petrelli, and Steve Whittaker. 2010. Sonic souvenirs: exploring the paradoxes of recorded sound for family remembering. In Proceedings of the 2010 ACM conference on Computer supported cooperative work (CSCW '10). ACM, New York, NY, USA, 391-400. DOI: https://doi.org/10.1145/1718918.1718985

6. Shogo Fukushima, Yuki Hashimoto, Takashi Nozawa, and Hiroyuki Kajimoto. 2010. Laugh enhancer using laugh track synchronized with the user's laugh motion. ACM Press, 3613.

7. John J. Godfrey and Edward Holliman. "Switchboard-1 Release 2." Linguistic Data Consortium, Philadelphia 926 (1997): 927.

8. Javier Hernandez, Mohammed (Ehsan) Hoque, Will Drevo, and Rosalind W. Picard. 2012. Mood meter: counting smiles in the wild. In Proceedings of the 2012 ACM Conference on Ubiquitous Computing (UbiComp '12). ACM, New York, NY, USA, 301-310. DOI=http://dx.doi.org/10.1145/2370216.2370264

9. Hayley Hung, Gwenn Englebienne, and Jeroen Kools. 2013. Classifying social actions with a single accelerometer. In Proceedings of the 2013 ACM international joint conference on Pervasive and ubiquitous computing (UbiComp '13). ACM, New York, NY, USA, 207-210. DOI=http://dx.doi.org/10.1145/2493432.2493513

10. Hiroshi Ishii, Ali Mazalek, and Jay Lee. 2001. Bottles as a minimal interface to access digital information. In CHI '01 Extended Abstracts on Human Factors in Computing Systems (CHI EA '01). ACM, New York, NY, USA, 187-188.

DOI=http://dx.doi.org/10.1145/634067.634180 
11. Jasmine Jones, David Merritt, and Mark S. Ackerman. 2017. KidKeeper: Design for Capturing Audio Mementos of Everyday Life for Parents of Young Children. In Proceedings of the 2017 ACM Conference on Computer Supported Cooperative Work and Social Computing (CSCW '17). ACM, New York, NY, USA, 1864-1875. DOI:

https://doi.org/10.1145/2998181.2998348

12. Lakshmish Kaushik, Abhijeet Sangwan, and John HL Hansen. "Laughter and filler detection in naturalistic audio." INTERSPEECH. 2015.

13. Rohit Ashok Khot, Ryan Pennings, and Florian "Floyd" Mueller. 2015. EdiPulse: Turning Physical Activity Into Chocolates. ACM Press, 331-334. http://doi.org/10.1145/2702613.2725436

14. Mary Tai Knox and Nikki Mirghafori. "Automatic laughter detection using neural networks." Interspeech. 2007.

15. Hyosun Kwon, Boriana Koleva, Holger Schnädelbach, and Steve Benford. 2017. "It's Not Yet A Gift": Understanding Digital Gifting. In Proceedings of the 2017 ACM Conference on Computer Supported Cooperative Work and Social Computing (CSCW '17). ACM, New York, NY, USA, 2372-2384. DOI: https://doi.org/10.1145/2998181.2998225

16. Sunmin Lee, (Wynnie) Wing Yi Chung, Emily Ip, and Thecla Schiphorst. 2014. The laughing dress: evoking prosocial interaction among strangers. In CHI '14 Extended Abstracts on Human Factors in Computing Systems (CHI EA '14). ACM, New York, NY, USA, 2143-2148. DOI: https://doi.org/10.1145/2559206.2581360

17. Diana L. Mahony, W. Jeffrey Burroughs, and Louis g. Lippman. 2002. Perceived Attributes of HealthPromoting Laughter: A Cross-Generational Comparison. The Journal of Psychology 136, 2: 171181. http://doi.org/10.1080/00223980209604148

18. Maurizio Mancini, Giovanna Varni, Radoslaw Niewiadomski, Gualtiero Volpe, and Antonio Camurri. 2014. How is your laugh today?. In CHI '14 Extended Abstracts on Human Factors in Computing Systems (CHI EA '14). ACM, New York, NY, USA, 1855 1860. DOI: https://doi.org/10.1145/2559206.2581205

19. Katsutoshi Masai, Yuta Sugiura, Katsuhiro Suzuki, Sho Shimamura, Kai Kunze, Masa Ogata, Masahiko Inami, and Maki Sugimoto. 2015. AffectiveWear: towards recognizing affect in real life. In Adjunct Proceedings of the 2015 ACM International Joint Conference on Pervasive and Ubiquitous Computing and Proceedings of the 2015 ACM International Symposium on Wearable Computers (UbiComp/ISWC'15 Adjunct). ACM, New York, NY, USA, 357-360. DOI: https://doi.org/10.1145/2800835.2800898
20. JA Meerloo. 1966. The biology of laughter. Psychoanalytic review 53, 2: 189-208.

21. Harold G. Nelson and Erik Stolterman (2003). Design Way: Intentional Change in an Unpredictable World. Educational Technology Publications Englewood Cliffs.

22. Michael Nunes, Saul Greenberg, and Carman Neustaedter. 2008. Sharing digital photographs in the home through physical mementos, souvenirs, and keepsakes. In Proceedings of the 7th ACM conference on Designing interactive systems (DIS '08). ACM, New York, NY, USA, 250-260. DOI=http://dx.doi.org/10.1145/1394445.1394472

23. William Odom, James Pierce, Erik Stolterman, and Eli Blevis. 2009. Understanding why we preserve some things and discard others in the context of interaction design. In Proceedings of the SIGCHI Conference on Human Factors in Computing Systems (CHI '09). ACM, New York, NY, USA, 1053-1062. DOI: https://doi.org/10.1145/1518701.1518862

24. William Odom, John Zimmerman, and Jodi Forlizzi. 2011. Teenagers and their virtual possessions: design opportunities and issues. In Proceedings of the SIGCHI Conference on Human Factors in Computing Systems (CHI '11). ACM, New York, NY, USA, 1491-1500. DOI: https://doi.org/10.1145/1978942.1979161

25. Daniela Petrelli and Steve Whittaker. 2010. Family memories in the home: contrasting physical and digital mementos. Personal Ubiquitous Comput. 14, 2 (February 2010), 153-169. DOI=http://dx.doi.org/10.1007/s00779-009-0279-7

26. Anne Marie Piper, Nadir Weibel, and James Hollan. 2013. Audio-enhanced paper photos: encouraging social interaction at age 105. In Proceedings of the 2013 conference on Computer supported cooperative work (CSCW '13). ACM, New York, NY, USA, 215224. DOI: https://doi.org/10.1145/2441776.2441802

27. Kimiko Ryokai, Elena Duran, Dina Bseiso, Noura Howell, and Ji Won Jun. 2017. Celebrating Laughter: Capturing and Sharing Tangible Representations of Laughter. In Proceedings of the 2017 ACM Conference Companion Publication on Designing Interactive Systems (DIS '17 Companion). ACM, New York, NY, USA, 202-206. DOI: https://doi.org/10.1145/3064857.3079146

28. Stefan Scherer, Michael Glodek, Friedhelm Schwenker, Nick Campbell, and Günther Palm. 2012. Spotting laughter in natural multiparty conversations: A comparison of automatic online and offline approaches using audiovisual data. ACM

29. Ayaka Shimasaki and Ryoko Ueoka. 2017. Laugh Log: E-textile Bellyband Interface for Laugh Logging. In Proceedings of the 2017 CHI Conference Extended Abstracts on Human Factors in Computing Systems 
(CHI EA '17). ACM, New York, NY, USA, 20842089. DOI: https://doi.org/10.1145/3027063.3053104

30. Alex S. Taylor and Richard Harper. 2003. The Gift of the Gab?: A Design Oriented Sociology of Young People's Use of Mobiles. Computer Supported Cooperative Work (CSCW) 12, 3: 267-296. http://doi.org/10.1023/A:1025091532662

31. Hitomi Tsujita and Jun Rekimoto. 2011. HappinessCounter: smile-encouraging appliance to increase positive mood. In CHI '11 Extended Abstracts on Human Factors in Computing Systems (CHI EA '11). ACM, New York, NY, USA, 117-126. DOI: https://doi.org/10.1145/1979742.1979608

32. Peter-Paul Verbeek, What Things Do: Philosophical Reflections on Technology, Agency and Design The Pennsylvania State University Press, University Park, Pennsylvania, 2005.
33. Yun Wang, Xiaojuan Ma, Qiong Luo, and Huamin Qu. 2016. Data Edibilization: Representing Data with Food. ACM Press, 409-422. http://doi.org/10.1145/2851581.2892570

34. Jun Wei, Xiaojuan Ma, and Shengdong Zhao. 2014. Food messaging: using edible medium for social messaging. ACM Press, 2873-2882. http://doi.org/10.1145/2556288.2557026

35. David West, Aaron Quigley, and Judy Kay. 2007. MEMENTO: a digital-physical scrapbook for memory sharing. Personal Ubiquitous Comput. 11, 4 (April 2007), 313-328.

DOI=http://dx.doi.org/10.1007/s00779-006-0090-7

36. Choc Edge: Welcome to the world of 3D chocolate printing! Retrieved March 19, 2017 from http://chocedge.com/ 